\title{
APPROACHABILITY AT THE SECOND SUCCESSOR OF A SINGULAR CARDINAL
}

\author{
MOTI GITIK AND JOHN KRUEGER
}

\begin{abstract}
We prove that if $\mu$ is a regular cardinal and $\mathbb{P}$ is a $\mu$-centered forcing poset, then $\mathbb{P}$ forces that $\left(I\left[\mu^{++}\right]\right)^{V}$ generates $I\left[\mu^{++}\right]$modulo clubs. Using this result, we construct models in which the approachability property fails at the successor of a singular cardinal. We also construct models in which the properties of being internally club and internally approachable are distinct for sets of size the successor of a singular cardinal.
\end{abstract}

In this paper we construct models in which the approachability property $\mathrm{AP}_{\mu^{+}}$ fails, where $\mu$ is a singular cardinal. We also obtain models where the properties of being internally club and internally approachable are distinct for models of size the successor of a singular cardinal. These results are related to the approachability ideal $I\left[\mu^{++}\right]$, where $\mu$ is a singular cardinal.

Theorem 1. Suppose $\mu$ is a regular cardinal and $\mathbb{P}$ is a $\mu$-centered forcing poset. Let $\lambda=\mu^{++}$. Then $\mathbb{P}$ forces that $(I[\lambda])^{V}$ generates $I[\lambda]$ modulo clubs.

Theorem 2. Suppose $\mu$ is supercompact and $\lambda>\mu$ is Mahlo.

(1) For any regular cardinal $\nu<\mu$, there is a forcing poset which forces that $\mu$ is a singular strong limit cardinal with cofinality $\nu$ and $A P_{\mu^{+}}$fails.

(2) For any limit ordinal $\alpha<\mu$, there is a forcing poset which forces that $\mu=\aleph_{\alpha}$ and $A P_{\mu^{+}}$fails.

Theorem 3. Suppose $\mu<\lambda$ are supercompact cardinals.

(1) For any regular $\nu<\mu$, there is a forcing poset which forces that $\mu$ is a singular strong limit cardinal with cofinality $\nu$, and for all regular $\theta \geq \mu^{++}$, there are stationarily many sets $N$ in $[H(\theta)]^{\mu^{+}}$which are internally club but not internally approachable.

(2) For any limit ordinal $\alpha<\mu$, there is a forcing poset which forces that $\mu=\aleph_{\alpha}$, and for all regular $\theta \geq \mu^{++}$, there are stationarily many sets $N$ in $[H(\theta)]^{\mu^{+}}$which are internally club but not internally approachable.

We assume that the reader has some basic familiarity with iterated forcing, generalized stationarity, generic elementary embeddings, and the interaction of forcing with elementary substructures ([1], [5]). For a cardinal $\mu$ and a set $X$ containing $\mu^{+}$, a set $S \subseteq[X]^{\mu^{+}}$is stationary if for any function $F:[X]^{<\omega} \rightarrow X$ there is $N$ in $S$ such that $\mu^{+} \subseteq N$ and $N$ is closed under $F$. This is equivalent to the property of having non-empty intersection with every closed and unbounded subset of $[X]^{\mu^{+}}$.

Date: January 2009.

The first author was partially supported by ISF Grant 234/08.

The second author would like to thank James Cummings for explaining the forcing poset from Section 4 for moving a supercompact cardinal $\mu$ with $2^{\mu}=\mu^{++}$down to $\aleph_{\omega}$ with a $\mu$-centered forcing poset. 
For a regular cardinal $\mu$ we let $\operatorname{ADD}(\mu)$ denote the Cohen forcing for adding a Cohen subset of $\mu$. If $\mu^{<\mu}=\mu, \operatorname{ADD}(\mu)$ has size $\mu$ and thus is $\mu^{+}$-c.c. For a regular cardinal $\kappa$ and $\lambda>\kappa$, we let $\operatorname{CoLL}(\kappa, \lambda)$ be the Lévy collapse for collapsing $\lambda$ to have size $\kappa$.

\section{The Approachability Ideal}

We review some well-known facts about the approachability ideal $I[\lambda]([15]$, [16]). Let $\lambda$ be a regular uncountable cardinal. Given a sequence $\vec{a}=\left\langle a_{i}: i<\lambda\right\rangle$ of bounded subsets of $\lambda$, let $S_{\vec{a}}$ denote the set of limit ordinals $\alpha<\lambda$ such that there exists a club set $c \subseteq \alpha$ with order type $\operatorname{cf}(\alpha)$ such that for all $\beta<\alpha$, there is $i<\alpha$ such that $c \cap \beta=a_{i}$. Let $I[\lambda]$ be the collection of sets $S \subseteq \lambda$ such that there exists $\vec{a}=\left\langle a_{i}: i<\lambda\right\rangle$, a sequence of bounded subsets of $\lambda$, and a club $C \subseteq \lambda$, such that $S \cap C \subseteq S_{\vec{a}}$. Then $I[\lambda]$ is a normal ideal on $\lambda$, which we refer to as the approachability ideal on $\lambda$.

Clearly if $A$ is in $I[\lambda]$, then for any set $S \subseteq \lambda$, if there is a club $C \subseteq \lambda$ such that $S \cap C \subseteq A$, then $S$ is in $I[\lambda]$. More generally, we say that a family $\mathcal{A} \subseteq I[\lambda]$ generates $I[\lambda]$ modulo clubs if $I[\lambda]$ is the collection of sets $S \subseteq \lambda$ such that there is a set $T$ in $\mathcal{A}$ and a club $C \subseteq \lambda$ such that $S \cap C \subseteq T$. Under some natural assumptions (for example, if $\lambda^{\overline{<\lambda}}=\lambda$ ), $I[\lambda]$ is generated by a single set modulo clubs. If $\{A\}$ generates $I[\lambda]$ modulo clubs, we say that $A$ generates $I[\lambda]$, and that $A$ is the maximal set in $I[\lambda]$. The maximal set, if it exists, is unique in the sense that if $A$ and $B$ both generate $I[\lambda]$, then there is a club set $C \subseteq \lambda$ such that $A \cap C=B \cap C$. It is an open problem whether $I[\lambda]$ must always have a maximal set.

In this paper we will be interested in the situation where $\lambda=\kappa^{+}$is a successor cardinal. In this case, $I[\lambda]$ is generated modulo clubs by sets of the form $S_{\vec{a}}$, where $\vec{a}=\left\langle a_{i}: i<\lambda\right\rangle$ is a sequence of subsets of $\lambda$ whose order types are less than $\kappa$. Assuming $\kappa^{<\kappa} \leq \kappa^{+}=\lambda, I[\lambda]$ has a maximal set. Namely, let $\vec{a}=\left\langle a_{i}: i<\lambda\right\rangle$ enumerate all subsets of $\lambda$ with order type less than $\kappa$. Then for any sequence $\vec{b}=\left\langle b_{i}: i\langle\lambda\rangle\right.$ of subsets of $\lambda$ with order type less than $\kappa$, there is a club $C \subseteq \lambda$ such that $C \cap S_{\vec{b}} \subseteq S_{\vec{a}}$. Hence $S_{\vec{a}}$ is the maximal set in $I[\lambda]$. In this case the maximal set $S_{\vec{a}}$ is stationary, and in fact, for all regular $\mu<\kappa^{+}, S_{\vec{a}} \cap \operatorname{cof}(\mu)$ is stationary (see Sections 8 and 9 of [2]).

For an uncountable cardinal $\kappa$, the approachability property at $\kappa$, written as $\mathrm{AP}_{\kappa}$, is the statement that $I\left[\kappa^{+}\right]$contains every subset of $\kappa^{+}$, or equivalently, $\kappa^{+}$is in $I\left[\kappa^{+}\right]$. In this case, there is a sequence $\vec{a}=\left\langle a_{i}: i<\kappa^{+}\right\rangle$consisting of sets in $\left[\kappa^{+}\right]^{<\kappa}$ such that $S_{\vec{a}}$ contains a club set. Clearly the failure of $\mathrm{AP}_{\kappa}$ is equivalent to the existence of a stationary subset of $\kappa^{+}$which is not in $I\left[\kappa^{+}\right]$.

It is a theorem of Shelah $([16])$ that if $\kappa$ is a regular uncountable cardinal, then $\kappa^{+} \cap \operatorname{cof}(<\kappa)$ is in $I\left[\kappa^{+}\right]$. So any stationary subset of $\kappa^{+}$which is not in $I\left[\kappa^{+}\right]$ concentrates on cofinality $\kappa$. In particular, if $I\left[\kappa^{+}\right]$has a maximal set $T$, then $T$ is equal modulo clubs to $\left(\kappa^{+} \cap \operatorname{cof}(<\kappa)\right) \cup(T \cap \operatorname{cof}(\kappa))$. So for a regular uncountable cardinal $\kappa, \mathrm{AP}_{\kappa}$ holds iff there is a sequence $\vec{a}=\left\langle a_{i}: i<\kappa^{+}\right\rangle$of subsets of $\kappa^{+}$with order type less than $\kappa$ and a club $C \subseteq \kappa^{+}$such that $C \cap \operatorname{cof}(\kappa) \subseteq S_{\vec{a}}$.

Let us describe another collection of sets which generates $I[\lambda]$ modulo clubs, where $\lambda$ is any uncountable regular cardinal. Fix a regular cardinal $\theta>\lambda$. Let $\vec{M}=$ $\left\langle M_{i}: i<\lambda\right\rangle$ be an increasing and continuous sequence of elementary substructures of $H(\theta)$ such that $\lambda \in M_{0}$, and for all $i<\lambda,\left|M_{i}\right|<\lambda, i \subseteq M_{i}$, and $M_{i} \cap \lambda \in \lambda$. 
Define $S_{\vec{M}}$ as the set of limit ordinals $\alpha<\lambda$ for which there exists a cofinal set $c \subseteq \alpha$ with order type $\operatorname{cf}(\alpha)$ such that for all $\beta<\alpha, c \cap \beta$ is in $M_{\alpha}$.

We claim that the collection of sets of the form $S_{\vec{M}}$ as above generates $I[\lambda]$ modulo clubs. First, let $\vec{M}=\left\langle M_{i}: i<\lambda\right\rangle$ be given, and we show $S_{\vec{M}}$ is in $I[\lambda]$. Let $\vec{a}=\left\langle a_{i}: i<\lambda\right\rangle$ enumerate $\bigcup\left\{M_{i}: i<\lambda\right\} \cap[\lambda]^{<\lambda}$. Then there is a club $D \subseteq \lambda$ such that for all $\beta$ in $D, \vec{a}\left\lceil\beta\right.$ enumerates $M_{\beta} \cap[\lambda]^{<\lambda}$. But then $S_{\vec{M}} \cap D \subseteq S_{\vec{a}}$, so $S_{\vec{M}}$ is in $I[\lambda]$. For if $\beta$ is in $S_{\vec{M}} \cap D$, fix a cofinal set $c \subseteq \beta$ with order type $\operatorname{cf}(\beta)$ whose proper initial segments are in $M_{\beta}$. Let $d$ be the closure of $c$. Then by elementarity, the initial segments of $d$ are in $M_{\beta}$ as well. So by choice of $D$, the initial segments of $d$ are in $\vec{a}\left\lceil\beta\right.$, and hence $\beta$ is in $S_{\vec{a}}$.

On the other hand, suppose $\vec{a}=\left\langle a_{i}: i<\lambda\right\rangle$ is given. Choose $\left\langle M_{i}: i<\lambda\right\rangle$ as above such that $\vec{a}$ is in $M_{0}$. Then $S_{\vec{a}} \subseteq S_{\vec{M}}$. For if $\beta$ is in $S_{\vec{a}}$, fix a club $c \subseteq \beta$ with order type $\operatorname{cf}(\beta)$ whose proper initial segments are in $\left\langle a_{i}: i<\beta\right\rangle$. Since $\beta \subseteq M_{\beta}$, by elementarity $\left\{a_{i}: i<\beta\right\} \subseteq M_{\beta}$. Hence every proper initial segment of $c$ is in $M_{\beta}$, so $\beta$ is in $S_{\vec{M}}$.

\section{Preserving the Approachability Ideal}

Let $\mu$ be a regular cardinal. A forcing poset $\mathbb{P}$ is said to be $\mu$-centered if $\mathbb{P}$ is the union of a family $\left\{A_{i}: i<\mu\right\}$ such that for all $i<\mu$, for all $p$ and $q$ in $A_{i}$, there is $r$ in $A_{i}$ such that $r \leq p, q$. Observe that any $\mu$-centered forcing poset $\mathbb{P}$ is $\mu^{+}$-c.c., since any subset of $\mathbb{P}$ of size $\mu^{+}$has $\mu^{+}$many elements in the same $A_{i}$. Also, any poset of size less than or equal to $\mu$ is $\mu$-centered, as can be seen by taking the $A_{i}$ 's to be singletons or empty.

Theorem 2.1. Suppose that $\mu$ is a regular cardinal and $\mathbb{P}$ is a $\mu$-centered forcing poset. Let $\lambda=\mu^{++}$. Then $\mathbb{P}$ forces that $(I[\lambda])^{V}$ generates $I[\lambda]$ modulo clubs. In particular, if $T$ is the maximal set in $I[\lambda]$, then $\mathbb{P}$ forces that $T$ remains the maximal set in $I[\lambda]$.

Proof. Fix a family $\left\{A_{i}: i<\mu\right\}$ witnessing that $\mathbb{P}$ is $\mu$-centered. Note that since $\mathbb{P}$ is $\mu^{+}$-c.c.,

$$
\left(\lambda \cap \operatorname{cof}\left(<\mu^{+}\right)\right)^{V}=\left(\lambda \cap \operatorname{cof}\left(<\mu^{+}\right)\right)^{V^{\mathbb{P}}} .
$$

Let $S_{<\mu^{+}}$denote this set. As pointed out in Section $1, S_{<\mu^{+}}$is in $I[\lambda]$, both in $V$ and $V^{\mathbb{P}}$. We also have that

$$
\left(\lambda \cap \operatorname{cof}\left(\mu^{+}\right)\right)^{V}=\left(\lambda \cap \operatorname{cof}\left(\mu^{+}\right)\right)^{V^{\mathbb{P}}} .
$$

First let us prove that $\mathbb{P}$ forces $(I[\lambda])^{V} \subseteq I[\lambda]$. So let $S$ be in $(I[\lambda])^{V}$. Fix a sequence $\vec{b}=\left\langle b_{i}: i\langle\lambda\rangle\right.$ of bounded subsets of $\lambda$ and a club $C \subseteq \lambda$ such that $S \cap C \subseteq S_{\vec{b}}$. We claim that in $V^{\mathbb{P}},\left(S_{\vec{b}}\right)^{V}$ is in $I[\lambda]$. It then follows that $S$ is in $I[\lambda]$ in $V^{\mathbb{P}}$ as well. On the one hand, $\left(S_{\vec{b}}\right)^{V} \cap \operatorname{cof}\left(<\mu^{+}\right) \subseteq S_{<\mu^{+}} \in I[\lambda]$. On the other hand, $\left(S_{\vec{b}}\right)^{V} \cap \operatorname{cof}\left(\mu^{+}\right) \subseteq\left(S_{\vec{b}}\right)^{V^{\mathbb{P}}} \in I[\lambda]$. For if $\alpha$ is in $S_{\vec{b}} \cap \operatorname{cof}\left(\mu^{+}\right)$in $V$, there is a club $c \subseteq \alpha$ with order type $\mu^{+}$whose initial segments are in $\vec{b}\lceil\alpha$. Since the cofinality of $\alpha$ remains $\mu^{+}$in $V^{\mathbb{P}}, \alpha$ is in $\left(S_{\vec{b}}\right)^{V^{\mathbb{P}}}$. Now $I[\lambda]$ is $\lambda$-complete, so $\left(S_{\vec{b}}\right)^{V}$ is in $I[\lambda]$ in $V^{\mathbb{P}}$.

Now we prove that $(I[\lambda])^{V}$ generates $I[\lambda]$ modulo clubs in $V^{\mathbb{P}}$. Let $\vec{a}=\left\langle\dot{a}_{i}: i<\right.$ $\lambda\rangle$ be a $\mathbb{P}$-name for a sequence of bounded subsets of $\lambda$. We will find a set $S$ in $I[\lambda]$ such that $\mathbb{P}$ forces $S_{\vec{a}} \subseteq S$. 
Fix a regular cardinal $\theta$ larger than $\lambda$ such that $\mathbb{P}$ is in $H(\theta)$. Choose an increasing and continuous sequence $\vec{M}=\left\langle M_{i}: i<\lambda\right\rangle$ of elementary substructures of $H(\theta)$ such that $\mu, \mathbb{P},\left\{A_{i}: i<\mu\right\}$, and $\vec{a}$ are in $M_{0}$, and for all $i<\lambda,\left|M_{i}\right|<\lambda, i \subseteq M_{i}$, and $M_{i} \cap \lambda \in \lambda$. Let $S_{\vec{M}}$ denote the set of limit ordinals $\alpha<\lambda$ such that there is a cofinal set $c \subseteq \alpha$ with order type $\operatorname{cf}(\alpha)$ such that every proper initial segment of $c$ is in $M_{\alpha}$. As noted in Section $1, S_{\vec{M}}$ is in $I[\lambda]$.

In $V^{\mathbb{P}}$ we have that $S_{\vec{a}} \cap S_{<\mu^{+}} \subseteq S_{<\mu^{+}}$, and $S_{<\mu^{+}}$is in $(I[\lambda])^{V}$. We will show that $S_{\vec{a}} \cap \operatorname{cof}\left(\mu^{+}\right) \subseteq\left(S_{\vec{M}}\right)^{V}$. Then $S_{\vec{a}} \subseteq S_{<\mu^{+}} \cup\left(S_{\vec{M}}\right)^{V} \in(I[\lambda])^{V}$, and we are done.

Let $\delta$ be in $\lambda \cap \operatorname{cof}\left(\mu^{+}\right)$, and suppose $p$ is a condition which forces $\delta$ is in $S_{\vec{a}}$. We will prove that $\delta$ is in $S_{\vec{M}}$. Choose a $\mathbb{P}$-name $\dot{c}$ for a club subset of $\delta$ with order type $\mu^{+}$such that $p$ forces every proper initial segment of $\dot{c}$ appears on the sequence $\left\langle\dot{a}_{i}: i<\delta\right\rangle$. For each $\alpha<\delta$, choose $r_{\alpha} \leq p$ and $\xi_{\alpha}<\delta$ such that

$$
r_{\alpha} \Vdash \dot{c} \cap \alpha=\dot{a}_{\xi_{\alpha}} .
$$

Now as $\mathbb{P}$ forces $\dot{c}$ has order type $\mu^{+}$and $\mathbb{P}$ is $\mu^{+}$-c.c., we can fix a club set $b \subseteq \delta$ with order type $\mu^{+}$such that $\mathbb{P}$ forces $b \subseteq \dot{c}$. For each $i<\mu$, let

$$
b_{i}=\left\{\alpha \in b: r_{\alpha} \in A_{i}\right\} .
$$

Since $b=\bigcup\left\{b_{i}: i<\mu\right\}$ and the order type of $b$ is $\mu^{+}$, we can fix $i<\mu$ such that $b_{i}$ is stationary in $\delta$. In particular, $b_{i}$ is cofinal in $\delta$.

Now define

$$
d=\left\{\nu<\delta: \exists r \in A_{i} r \Vdash \nu \in \dot{c}\right\} .
$$

Claim. The set $d$ is a cofinal subset of $\delta$ with order type $\mu^{+}$, all of whose initial segments are in $M_{\delta}$. Therefore $\delta$ is in $S_{\vec{M}}$.

Proof. Note that $b \subseteq d$, so $d$ is cofinal in $\delta$. If the order type of $d$ is greater than $\mu^{+}$, then there is $\alpha<\delta$ such that $|d \cap \alpha| \geq \mu^{+}$. Since $\mathbb{P}$ forces $\dot{c} \cap \alpha$ has size $\mu$, by the $\mu^{+}$-c.c. of $\mathbb{P}$ there is a set $a$ with size $\mu$ such that $\mathbb{P}$ forces $\dot{c} \cap \alpha \subseteq a$. But then $d \cap \alpha \subseteq a$, which is a contradiction. For if $\nu$ is in $d \cap \alpha$, there is $r$ in $A_{i}$ which forces that $\nu$ is in $\dot{c} \cap \alpha$. So $r$ forces $\nu$ is in $a$, and hence $\nu$ actually is in $a$.

Now we prove that for all $\gamma<\delta, d \cap \gamma$ is in $M_{\delta}$. So fix $\gamma<\delta$. Choose $\alpha$ in $b_{i}$ which is larger than $\gamma$. We will show $d \cap \alpha$ is in $M_{\delta}$. Then since $\delta \subseteq M_{\delta}, \gamma$ is in $M_{\delta}$, so by elementarity $d \cap \gamma=(d \cap \alpha) \cap \gamma$ is in $M_{\delta}$.

To prove that $d \cap \alpha$ is in $M_{\delta}$, we show that

$$
d \cap \alpha=\left\{\nu<\alpha: \exists r \in A_{i} r \Vdash \nu \in \dot{a}_{\xi_{\alpha}}\right\} .
$$

Then since $\delta \subseteq M_{\delta}$, the objects $\alpha, \xi_{\alpha}, \dot{a}_{\xi_{\alpha}}$, and $A_{i}$ are all in $M_{\delta}$, so by elementarity $d \cap \alpha$ is in $M_{\delta}$.

Assume on the one hand that $\nu$ is in $d \cap \alpha$. Then by definition of $d$, there is $r$ in $A_{i}$ which forces $\nu$ is in $\dot{c}$. Since $\alpha$ is in $b_{i}, r_{\alpha}$ is in $A_{i}$. So we can fix $s \leq r, r_{\alpha}$ in $A_{i}$. Since $s \leq r_{\alpha}, s \Vdash \dot{c} \cap \alpha=\dot{a}_{\xi_{\alpha}}$. But since $s \leq r, s \Vdash \nu \in \dot{c} \cap \alpha$. So $s \Vdash \nu \in \dot{a}_{\xi_{\alpha}}$, as desired. Suppose on the other hand that $\nu<\alpha$ and there is $r$ in $A_{i}$ which forces $\nu$ is in $\dot{a}_{\xi_{\alpha}}$. Again choose $s \leq r, r_{\alpha}$ in $A_{i}$. Then $s$ forces $\dot{c} \cap \alpha=\dot{a}_{\xi_{\alpha}}$ and $\nu \in \dot{a}_{\xi_{\alpha}}$. So $s$ forces $\nu$ is in $\dot{c} \cap \alpha$. By definition of $d, \nu$ is in $d \cap \alpha$, and we are finished with the proof of the claim.

Suppose that $T$ is the maximal set in $I[\lambda]$. Let $G$ be a generic filter for $\mathbb{P}$ over $V$, and we show that $T$ is maximal in $I[\lambda]$ in $V[G]$. Working in $V[G]$, let $S$ be in $I[\lambda]$. Since $(I[\lambda])^{V}$ generates $I[\lambda]$ modulo clubs in $V[G]$, there is a set $A$ in $(I[\lambda])^{V}$ 
and a club $C \subseteq \lambda$ in $V[G]$ such that $S \cap C \subseteq A$. Since $T$ is maximal in $V$, there is a club $D \subseteq \lambda$ such that $A \cap D \subseteq T$. Then $S \cap C \cap D \subseteq A \cap D \subseteq T$.

Corollary 2.2. Suppose $\mu$ is a regular cardinal and $\mathbb{P}$ is a $\mu$-centered forcing poset. Let $\lambda=\mu^{++}$. If $S \subseteq \lambda$ is a stationary set which is not in $I[\lambda]$, then $\mathbb{P}$ forces $S$ is not in $I[\lambda]$. In particular, if $A P_{\mu^{+}}$fails, then $\mathbb{P}$ forces $A P_{\mu^{+}}$fails.

Proof. Let $S$ be a stationary subset of $\lambda$. Let $G$ be a generic filter for $\mathbb{P}$ over $V$. Suppose in $V[G]$ that $S$ is in $I[\lambda]$. Then by Theorem 2.1, there is a club $C \subseteq \lambda$ and a set $A$ in $(I[\lambda])^{V}$ such that $S \cap C \subseteq A$. Since $\mathbb{P}$ is $\mu^{+}$-c.c., there is a club $D \subseteq C$ in $V$. Then $S \cap D \subseteq A$, so $S$ is in $(I[\lambda])^{V}$.

Now if $\mathrm{AP}_{\mu^{+}}$fails, then there is a stationary set $S \subseteq \lambda$ which is not in $I[\lambda]$. In $V^{\mathbb{P}}, S$ remains stationary, and $S$ is still not in $I[\lambda]$ by what we just proved. So $\mathrm{AP}_{\mu^{+}}$fails in $V^{\mathbb{P}}$.

\section{Failure of the Approachability Property}

We present now a general construction of a model in which the approachability property $\mathrm{AP}_{\kappa}$ fails, where $\kappa$ is a regular cardinal. The construction is essentially an iterated forcing version of the Mitchell model with no special Aronszajn trees on $\kappa^{+}([13])$. The fact that the approachability property fails in Mitchell's model is well-known.

The forcing iteration we use both in this section and in Section 5 is a special case of the mixed support iterated forcing schema presented in [8]. This iteration schema is described in the next theorem.

Theorem 3.1 ([8], [6]). Suppose $\mu^{<\mu}=\mu, \kappa$ is a regular cardinal greater than $\mu$, and for all $\nu<\kappa, \nu^{<\mu}<\kappa$. Consider a forcing iteration

$$
\left\langle\mathbb{P}_{i}, \dot{\mathbb{Q}}_{j}: i \leq \alpha, j<\alpha\right\rangle
$$

satisfying the following properties:

(1) If $i<\alpha$ is even, then $\mathbb{P}_{i}$ forces $\dot{\mathbb{Q}}_{i}=\operatorname{ADD}(\mu)$.

(2) If $i<\alpha$, then $\mathbb{P}_{i}$ forces that $\dot{\mathbb{Q}}_{i}$ is $\mu$-closed.

(3) If $\delta \leq \alpha$ is a limit ordinal, then $\mathbb{P}_{\delta}$ consists of all partial functions $p: \delta \rightarrow V$ such that $p\left\lceil i\right.$ is in $\mathbb{P}_{i}$ for all $i<\delta, \mid \operatorname{dom}(p) \cap$ Even $\mid<\mu$, and $|\operatorname{dom}(p) \cap \operatorname{Odd}|<\kappa$. For $p$ and $q$ in $\mathbb{P}_{\delta}$, let $q \leq p$ if $\operatorname{dom}(p) \subseteq \operatorname{dom}(q)$ and for all $i$ in $\operatorname{dom}(p), q\lceil i$ forces $q(i) \leq p(i)$.

In addition, for all even $i$ with $i+1<\alpha$, define a weak ordering $\leq^{*}$ on $\operatorname{ADD}(\mu) *$ $\dot{\mathbb{Q}}_{i+1}$ in $V^{\mathbb{P}_{i}}$ by letting $p_{2} * \dot{q}_{2} \leq^{*} p_{1} * \dot{q}_{1}$ if $p_{2} * \dot{q}_{2} \leq p_{1} * \dot{q}_{1}$ and $p_{2}=p_{1}$.

(4) If $i$ is even and $i+1<\alpha$, then $\mathbb{P}_{i}$ forces that $\left\langle\operatorname{ADD}(\mu) * \dot{\mathbb{Q}}_{i+1}, \leq^{*}\right\rangle$ is $\kappa$-strategically closed.

Assuming that these requirements are satisfied, the iteration has the property that for all even $i$ with $i+1<\alpha, \mathbb{P}_{\alpha}$ factors as $\mathbb{P}_{i} * \mathbb{P}_{i, \alpha}$, such that in $V^{\mathbb{P}_{i}}$ :

(I) $\mathbb{P}_{i, \alpha}$ preserves all cardinals and cofinalities less than or equal to $\kappa$.

(II) $\mathbb{P}_{i, \alpha}$ forces that whenever $f: \kappa \rightarrow V^{\mathbb{P}_{i}}$ is a function, all of whose proper initial segments are in $V^{\mathbb{P}_{i}}$, then $f$ is in $V^{\mathbb{P}_{i}}$. 
Of course the case $i=0$ and $\mathbb{P}_{i, \alpha}=\mathbb{P}_{\alpha}$ in the conclusion of the theorem is of special importance. This case was proven in [8]. The proof of the general statement of the theorem, which is given in Theorem 8.3 of [6], combines the material of [8] with the usual intermediate stage analysis of forcing iterations.

Assume for the rest of this section that $\mu$ is a regular cardinal such that $\mu^{<\mu}=\mu$, $\kappa$ is a regular cardinal greater than $\mu$, and for all $\nu<\kappa, \nu^{<\mu}<\kappa$. Also assume that $\lambda>\kappa$ is a Mahlo cardinal.

We describe a natural model in which the approachability property on $\kappa$ fails. We define by recursion a forcing iteration

$$
\left\langle\mathbb{P}_{i}, \dot{\mathbb{Q}}_{j}: i \leq \lambda, j<\lambda\right\rangle .
$$

Suppose $i<\lambda$ and $\mathbb{P}_{i}$ is defined. If $i$ is even, let $\dot{\mathbb{Q}}_{i}$ be a $\mathbb{P}_{i}$-name for $\operatorname{ADD}(\mu)$. If $i$ is odd, let $\dot{\mathbb{Q}}_{i}$ be a $\mathbb{P}_{i}$-name for $\operatorname{CoLL}\left(\kappa, \kappa^{+}\right)$. For even ordinals $i<\alpha$, define a weak ordering $\leq^{*}$ on $\dot{\mathbb{Q}}_{i} * \dot{\mathbb{Q}}_{i+1}=\operatorname{ADD}(\mu) * \operatorname{CoLL}\left(\kappa, \kappa^{+}\right)$in $V^{\mathbb{P}_{i}}$ by letting $p_{2} * \dot{q}_{2} \leq^{*} p_{1} * \dot{q}_{1}$ if $p_{2} * \dot{q}_{2} \leq p_{1} * \dot{q}_{1}$ and $p_{2}=p_{1}$. Clearly $\left\langle\dot{\mathbb{Q}}_{i} * \dot{\mathbb{Q}}_{i+1}, \leq^{*}\right\rangle$ is $\kappa$-closed, and hence $\kappa$ strategically closed, in $V^{\mathbb{P}_{i}}$.

Suppose $\delta \leq \lambda$ is a limit ordinal and for all $i<\delta, \mathbb{P}_{i}$ is defined. A condition in $\mathbb{P}_{\delta}$ is a partial function $p: \delta \rightarrow V$ such that for all $i<\delta, p\left\lceil i\right.$ is in $\mathbb{P}_{i}, \mid \operatorname{dom}(p) \cap$ Even $\mid<$ $\mu$, and $|\operatorname{dom}(p) \cap \operatorname{Odd}|<\kappa$. Define $q \leq p$ in $\mathbb{P}_{\delta}$ if $\operatorname{dom}(p) \subseteq \operatorname{dom}(q)$, and for all $i$ in $\operatorname{dom}(p), q\lceil i \Vdash q(i) \leq p(i)$.

This iteration clearly satisfies the hypotheses of Theorem 3.1. Hence properties (I) and (II) in the conclusion of the theorem hold.

Define a stationary set $A \subseteq \lambda$ by letting

$$
A=\{\alpha<\lambda: \kappa<\alpha \wedge \alpha \text { is strongly inaccessible }\} .
$$

Consider an ordinal $\alpha$ in $A \cup\{\lambda\}$. Then for all $\beta<\alpha,\left|\mathbb{P}_{\beta}\right|<\alpha$. Moreover, for any ordinal $\delta \leq \alpha$ with cofinality greater than or equal to $\kappa, \mathbb{P}_{\delta}$ is the direct limit of $\left\langle\mathbb{P}_{i}: i<\delta\right\rangle$. By standard arguments, this implies $\mathbb{P}_{\alpha}$ is $\alpha$-c.c. Since we use Lévy collapses in the iteration, $\mathbb{P}_{\alpha}$ forces that $\alpha$ is equal to $\kappa^{+}$. Also by a standard argument, any bounded subset of $\alpha$ in $V^{\mathbb{P}_{\alpha}}$ is in $V^{\mathbb{P}_{i}}$ for some $i<\alpha$. In particular, it follows that $\mathbb{P}_{\lambda}$ forces that $\lambda=\kappa^{+}$and $2^{\mu}=\lambda$.

We also note that if $\alpha$ is in $A$, then $\mathbb{P}_{\lambda}$ forces that $\alpha$ has cofinality $\kappa$. Indeed, factor $\mathbb{P}_{\lambda}$ as

$$
\mathbb{P}_{\lambda}=\mathbb{P}_{\alpha} * \operatorname{ADD}(\mu) * \operatorname{CoLL}\left(\kappa, \kappa^{+}\right) * \mathbb{P}_{\alpha+2, \lambda} .
$$

In $V^{\mathbb{P}_{\alpha}}, \alpha$ is equal to $\kappa^{+}$as noted above. So after forcing over $V^{\mathbb{P}_{\alpha}}$ with $\operatorname{ADD}(\mu) *$ $\operatorname{Colv}\left(\kappa, \kappa^{+}\right), \alpha$ has cofinality $\kappa$. But $\mathbb{P}_{\alpha+2, \lambda}$ preserves the regularity of $\kappa$ by property (I). So in $V^{\mathbb{P}_{\lambda}}, \alpha$ has cofinality $\kappa$.

Proposition 3.2. The poset $\mathbb{P}_{\lambda}$ forces that $I[\lambda]$ does not contain any stationary subset of $A$. In particular, $\mathbb{P}_{\lambda}$ forces that $A$ is not in $I[\lambda]$, so $A P_{\kappa}$ fails.

Proof. Let $G$ be a generic filter for $\mathbb{P}_{\lambda}$ over $V$. For all $i<\lambda$, let $G_{i}=G \cap \mathbb{P}_{i}$. Note that since $\mathbb{P}_{\lambda}$ is $\lambda$-c.c., $A$ remains stationary in $V[G]$. Let $B$ be a stationary subset of $A$ in $V[G]$. Suppose for a contradiction that there is a sequence $\vec{a}=\left\langle a_{i}: i<\lambda\right\rangle$ of bounded subsets of $\lambda$ and a club $C \subseteq \lambda$ such that $B \cap C \subseteq S_{\vec{a}}$.

Define $F: \lambda \rightarrow \lambda$ as follows. For $i<\lambda, a_{i}$ is a bounded subset of $\lambda$, and therefore $a_{i}$ is in $V\left[G_{j}\right]$ for some $j<\lambda$. Let $F(i)$ be the least $j<\lambda$ such that $a_{i}$ is in $V\left[G_{j}\right]$. Let $D$ be the club set of $\alpha<\lambda$ such that for all $i<\alpha, F(i)<\alpha$.

Fix $\delta$ in $B \cap C \cap D$. Then $\delta$ is in $S_{\vec{a}}$. Fix a club set $c \subseteq \delta$ with order type equal to the cofinality of $\delta$, which is $\kappa$, such that for all $\beta<\delta, c \cap \beta$ is equal to $a_{i_{\beta}}$ for 
some $i_{\beta}<\delta$. Then for all $\beta<\delta, c \cap \beta$ is in $V\left[G_{F\left(i_{\beta}\right)}\right]$. Since $\delta$ is in $D, F\left(i_{\beta}\right)<\delta$, so $c \cap \beta$ is in $V\left[G_{\delta}\right]$.

Now factor $\mathbb{P}_{\lambda}$ as $\mathbb{P}_{\delta} * \mathbb{P}_{\delta, \lambda}$. By property (II), $\mathbb{P}_{\delta, \lambda}$ forces over $V\left[G_{\delta}\right]$ that any function $f: \kappa \rightarrow O n$, all of whose initial segments are in $V\left[G_{\delta}\right]$, must itself be in $V\left[G_{\delta}\right]$. Let $f: \kappa \rightarrow O n$ be the unique order preserving bijection from $\kappa$ onto $c$. Since every proper initial segment of $c$ is in $V\left[G_{\delta}\right]$, clearly every proper initial segment of $f$ is in $V\left[G_{\delta}\right]$ as well. So $f$ is in $V\left[G_{\delta}\right]$. But this implies that $\delta$ has cofinality $\kappa$ in $V\left[G_{\delta}\right]$. This is impossible, since $\mathbb{P}_{\delta}$ forces that $\delta$ is equal to $\kappa^{+}$.

Note that in $V^{\mathbb{P}_{\lambda}}$ there are no special Aronszajn trees on $\kappa^{+}$. Indeed, the existence of a special Aronszajn tree on $\kappa^{+}$is equivalent to weak square $\square_{\kappa}^{*}$. But $\square_{\kappa}^{*}$ implies $\mathrm{AP}_{\kappa}$. Since $\mathrm{AP}_{\kappa}$ fails in $V^{\mathbb{P}_{\lambda}}$, so does $\square_{\kappa}^{*}$. For proofs of these comments, see $[2]$.

\section{Approachability at the Second Successor of a Singular}

We now show how to obtain a failure of the approachability property $\mathrm{AP}_{\mu^{+}}$, where $\mu$ is a singular cardinal. The general idea is to force a model where $\mathrm{AP}_{\mu^{+}}$ fails using the method of the previous section, while maintaining that $\mu$ is a large cardinal. Then apply Corollary 2.2 to forcing posets which singularize $\mu$, such as Prikry forcing.

Let $\mathbb{P}$ be a forcing poset. A set $A \subseteq \mathbb{P}$ is said to be directed if for all $p$ and $q$ in $A$, there is $r$ in $A$ such that $r \leq p, q$. For a regular cardinal $\mu, \mathbb{P}$ is $\mu$-directed closed if any directed subset of $\mathbb{P}$ with size less than $\mu$ has a lower bound.

Lemma 4.1. Suppose $\mu^{<\mu}=\mu, \kappa$ is a regular cardinal larger than $\mu$, and for all $\nu<\kappa, \nu^{<\mu}<\kappa$. Let $\left\langle\mathbb{P}_{i}, \dot{\mathbb{Q}}_{j}: i \leq \alpha, j<\alpha\right\rangle$ be a forcing iteration satisfying the hypotheses of Theorem 3.1. Assume in addition that for all $i<\alpha, \mathbb{P}_{i}$ forces that $\dot{\mathbb{Q}}_{i}$ is $\mu$-directed closed. Then $\mathbb{P}_{\alpha}$ is $\mu$-directed closed.

The proof is essentially the same as the standard proof that an iteration of $\mu$ directed closed forcings is $\mu$-directed closed, provided that the iteration uses only direct and inverse limits, and uses inverse limits at any stage of cofinality less than $\mu$. See, for example, Lemma 21.7 of [5].

Now we describe our forcing construction. Suppose that $\mu$ is a supercompact cardinal. Assume moreover that the supercompactness of $\mu$ is indestructible under $\mu$-directed closed forcing. To obtain this, start with a model in which $\mu$ is supercompact, and force with the $\mu$-c.c. forcing poset of size $\mu$ as described in [10] to obtain a generic extension in which $\mu$ is indestructible.

Assume that $\lambda$ is a Mahlo cardinal larger than $\mu$. Let $\left\langle\mathbb{P}_{i}, \dot{\mathbb{Q}}_{j}: i \leq \lambda, j<\lambda\right\rangle$ be the forcing iteration described in the previous section, where $\kappa=\mu^{+}$. So for even $i<\lambda, \dot{\mathbb{Q}}_{i}$ is a name for $\operatorname{ADD}(\mu)$, and for odd $i<\lambda, \dot{\mathbb{Q}}_{i}$ is a name for $\operatorname{CoLL}\left(\mu^{+}, \mu^{++}\right)$. Clearly for all $i<\lambda, \dot{\mathbb{Q}}_{i}$ is forced to be $\mu$-directed closed. So $\mathbb{P}_{\lambda}$ is $\mu$-directed closed by Lemma 4.1 .

Let $G$ be a generic filter for $\mathbb{P}_{\lambda}$ over $V$, and let $W=V[G]$. Then in $W, \mu$ remains a supercompact cardinal, $\lambda=\mu^{++}=2^{\mu}$, and the approachability property $\mathrm{AP}_{\mu^{+}}$fails. By Corollary 2.2, any forcing poset in $W$ which is $\mu$-centered forces that $\mathrm{AP}_{\mu^{+}}$fails.

Since $\mu$ is supercompact in $W$, it is measurable. Fix a normal ultrafilter $U$ on $\mu$. Recall the Prikry forcing $\mathbb{P}$ defined from $U$ in $W$. A condition in $\mathbb{P}$ is a pair $\langle a, A\rangle$, where $a$ is a finite subset of $\mu, A$ is in $U$, and for all $\alpha$ in $A, \alpha>\max (a)$. The 
ordering on $\mathbb{P}$ is defined by letting $\langle b, B\rangle \leq\langle a, A\rangle$ if $b \cap(\max (a)+1)=a, B \subseteq A$, and $b \backslash a \subseteq A$. As is well known, $\mathbb{P}$ adds a cofinal $\omega$-sequence to $\mu$, and does not add any bounded subsets to $\mu$. So in $V^{\mathbb{P}}, \mu$ is a singular strong limit cardinal with cofinality $\omega$. By Corollary 2.2 and the next lemma, $\mathrm{AP}_{\mu^{+}}$fails in $V^{\mathbb{P}}$.

Lemma 4.2. The Prikry forcing $\mathbb{P}$ is $\mu$-centered.

Proof. Let $\left\langle a_{i}: i<\mu\right\rangle$ enumerate $[\mu]^{<\omega}$, and for each $i<\mu$, define $A_{i}=\{\langle a, A\rangle$ : $\left.a=a_{i}\right\}$. Then if $\left\langle a_{i}, A\right\rangle$ and $\left\langle a_{i}, B\right\rangle$ are both in $A_{i}$, the condition $\left\langle a_{i}, A \cap B\right\rangle$ is a common refinement of both conditions which is also in $A_{i}$.

Since $\mu$ is a supercompact cardinal in $W$, there exist coherent sequences of normal ultrafilters on $\mu$ of any length up to $\left(2^{\mu}\right)^{+}$. From such sequences we can define Magidor forcing [12] and Radin forcing [14]. Both Magidor forcing and Radin forcing on $\mu$ are $\mu$-centered, by an easy argument similar to the proof of Lemma 4.2. So in particular, for any regular $\nu<\mu$, if $\mathbb{Q}$ is the Magidor forcing using a coherent sequence of ultrafilters of length $\nu$, then $\mathbb{Q}$ preserves all cardinals, forces that $\mu$ is a singular strong limit cardinal with cofinality $\nu$, and forces that $\mathrm{AP}_{\mu^{+}}$ fails.

Let us now discuss the failure of the approachability property at $\aleph_{\omega+1}$. We use the following fact, which we will discuss in more detail momentarily.

Theorem 4.3 ([11], [4]). Suppose that $\mu$ is $\mu^{++}$-supercompact and $2^{\mu}=\mu^{++}$. Then there exists a $\mu$-centered forcing poset $\mathbb{Q}$ which collapses $\mu$ to become $\aleph_{\omega}$.

In the model $W, \mu$ is supercompact, $2^{\mu}=\mu^{++}$, and $\mathrm{AP}_{\mu^{+}}$fails. Using Theorem 4.3 , let $\mathbb{Q}$ be a $\mu$-centered forcing poset which collapses $\mu$ to become $\aleph_{\omega}$. Let $K$ be a generic filter for $\mathbb{Q}$ over $W$. Then by Corollary $2.2, \mathrm{AP}_{\mu^{+}}$fails in $W[K]$. But $\mu^{+}$ is equal to $\aleph_{\omega+1}$ in $W[K]$. So $\mathrm{AP}_{\aleph_{\omega+1}}$ fails in this model.

The proof of Theorem 4.3 is beyond the scope of this paper. But we will give the definition of the forcing and explain why it is $\mu$-centered. To define the poset we only use the assumptions that $\mu$ is supercompact and $2^{\mu}=\mu^{++}$in $W$.

Let $U$ be a normal ultrafilter on $P_{\mu}\left(\mu^{++}\right)$. Let $j: V \rightarrow M=U l t(V, U)$ be the ultrapower map. Let $U_{0}$ be the restriction of $U$ to $\mu$, that is, $U_{0}$ is the collection of sets $A \subseteq \mu$ such that $\mu \in j(A)$. Let $j_{0}: V \rightarrow M_{0}=U l t\left(V, U_{0}\right)$ be the ultrapower map.

Let $X$ denote the set of strongly inaccessible cardinals below $\mu$. For each $\alpha$ in $X$, let $\mathcal{B}_{\alpha}$ denote the Boolean completion of $\operatorname{CoLL}\left(\alpha^{+3},<\mu\right)$. Then the map $\alpha \mapsto \mathcal{B}_{\alpha}$ represents in $M_{0}$ the Boolean completion of $\operatorname{CoLL}\left(\mu^{+3},<j_{0}(\mu)\right)$, which we denote by $\mathcal{B}_{0}$.

To define $\mathbb{Q}$, we use the following fact, which we quote without proof: there exists a $\mu$-complete ultrafilter $\mathcal{F}$ on $\mathcal{B}_{0}$. That is, there is a filter $\mathcal{F} \subseteq \mathcal{B}_{0}$ consisting of non-zero conditions such that for any $b$ in $\mathcal{B}_{0}$, either $b$ or $-b$ is in $\mathcal{F}$, and if $A \subseteq \mathcal{F}$ is a family of fewer than $\mu$ many elements of $\mathcal{F}$, there is $c$ in $\mathcal{F}$ such that $c \leq b$ for all $b$ in $\mathcal{F}$.

Define a forcing poset $\mathbb{Q}$ as follows. A condition in $\mathbb{Q}$ is a sequence

$$
\left\langle f, \alpha_{0}, f_{0}, \ldots, \alpha_{n-1}, f_{n-1}, A, h\right\rangle
$$

such that:

(1) $\alpha_{0}<\ldots<\alpha_{n-1}$ are in $X$,

(2) if $n=0$ then $f$ is in $\operatorname{ColL}\left(\omega_{3},<\mu\right)$, and if $n>0$ then $f$ is in $\operatorname{CoLL}\left(\omega_{3},<\alpha_{0}\right)$, 
(3) $f_{n-1}$ is in $\operatorname{CoLL}\left(\alpha_{n-1}^{+3},<\mu\right)$,

(4) for $i<n-1, f_{i}$ is in $\operatorname{CoLL}\left(\alpha_{i}^{+3},<\alpha_{i+1}\right)$,

(5) $A \subseteq X$ is in $U_{0}$, and for all $\alpha$ in $A, \alpha_{n-1}<\alpha$, and $\operatorname{ran}\left(f_{n-1}\right) \subseteq \alpha$,

(6) $h: A \rightarrow V$ is a function such that for all $\alpha$ in $A, h(\alpha)$ is in $\mathcal{B}_{\alpha}$,

(7) $j_{0}(h)(\mu) \in \mathcal{F}$.

We define the ordering on $\mathbb{Q}$ as follows. Suppose we have conditions

$$
p=\left\langle f, \alpha_{0}, f_{0}, \ldots, \alpha_{n-1}, f_{n-1}, A, h\right\rangle
$$

and

Let $q \leq p$ if:

$$
q=\left\langle g, \beta_{0}, g_{0}, \ldots, \beta_{m-1}, g_{m-1}, B, k\right\rangle
$$

(a) $m \geq n$,

(b) for all $i<n, \alpha_{i}=\beta_{i}$,

(c) $f \subseteq g$, and for all $i<n, f_{i} \subseteq g_{i}$,

(d) $B \subseteq A$,

(e) for all $\alpha$ in $B, k(\alpha) \leq h(\alpha)$,

(f) for $n \leq j<m, \beta_{j}$ is in $A$,

(g) for $n \leq j<m, g_{j}$ is a refinement of $h\left(\beta_{j}\right)$.

Let $q \leq^{*} p$ if $q \leq p$ and $m=n$.

Lemma 4.4. The forcing poset $\mathbb{Q}$ is $\mu$-centered.

Proof. Let $\left\langle t_{i}: i<\mu\right\rangle$ enumerate all sequences $t=\left\langle f, \alpha_{0}, f_{0}, \ldots, \alpha_{n-1}, f_{n-1}\right\rangle$ such that for some $A$ and $h,\langle t, A, h\rangle$ is a condition in $\mathbb{Q}$. For each $i<\mu$ define $A_{i}$ as the collection of conditions $p$ of the form $p=\left\langle t_{i}, A, h\right\rangle$.

Suppose $\left\langle t_{i}, A, h\right\rangle$ and $\left\langle t_{i}, B, k\right\rangle$ are both in $A_{i}$. Since $j_{0}(h)(\mu)$ and $j_{0}(k)(\mu)$ are in $\mathcal{F}$ and $\mathcal{F}$ is a filter, there is $b$ in $\mathcal{F}$ such that $b \leq j_{0}(h)(\mu), j_{0}(k)(\mu)$. Since $b$ is in $\mathcal{B}_{0}$, we can fix a function $f: X \rightarrow V$ such that $f(\alpha) \in \mathcal{B}_{\alpha}$ for all $\alpha$ in $X$ and $f$ represents $b$ in $M_{0}$, that is, $j_{0}(f)(\mu)=b$. Since $b \leq j_{0}(h)(\mu), j_{0}(k)(\mu)$, there is a set $C$ in $U_{0}$ such that for all $\alpha$ in $C, f(\alpha) \leq h(\alpha), k(\alpha)$. Then $\left\langle t_{i}, A \cap B \cap C, f \uparrow A \cap B \cap C\right\rangle$ is a condition in $A_{i}$ below $\left\langle t_{i}, A, h\right\rangle$ and $\left\langle t_{i}, B, k\right\rangle$.

The poset $\mathbb{Q}$ just described adds a cofinal $\omega$-sequence of regular cardinals to $\mu$, and Lévy collapses all but finitely many cardinals in between successive cardinals in the sequence. By using a coherent sequence of ultrafilters in place of a single ultrafilter, we can generalize the poset $\mathbb{Q}$ to posets which can add a cofinal sequence to $\mu$ of any limit order type less than $\mu$, while Lévy collapsing all but finitely many cardinals in between successive cardinals in the sequence. This Magidor forcing analogue of the poset $\mathbb{Q}$ above is also $\mu$-centered, by the same argument as in Lemma 4.4. Thus in $W$, for any limit ordinal $\alpha<\mu$, there is a $\mu$-centered forcing poset which collapses $\mu$ to become $\aleph_{\alpha}$, while preserving the failure of $\mathrm{AP}_{\mu^{+}}$. This completes the proof of Theorem 2.

\section{Internally Club and Internally Approachable}

Consider regular cardinals $\kappa<\lambda$. We say that a set $N \prec H(\lambda)$ with size $\kappa$ is internally approachable if $N$ is the union of an increasing and continuous sequence $\left\langle N_{i}: i<\kappa\right\rangle$ such that for all $\alpha<\kappa,\left\langle N_{i}: i<\alpha\right\rangle$ is in $N$. The set $N$ is internally club if $N$ is the union of an increasing and continuous sequence $\left\langle N_{i}: i<\kappa\right\rangle$ such that for all $i<\kappa, N_{i}$ is in $N$. 
Foreman and Todorčević [3] asked whether the properties of being internally approachable and internally club are equivalent. The second author solved this problem in the negative in [7], in the case of sets of size $\aleph_{1}$; this result was generalized in [9] and [6] to sets of size the successor of a regular cardinal, and to sets of size an inaccessible cardinal. In this section we complete the solution to this problem by handling the case of sets of size the successor of a singular cardinal.

A general construction of a model in which internally club and internally approachable are distinct is given in Section 11 of [6]. Rather than repeating this construction in full detail here, we will black box several facts and refer the reader to [6] for the complete details. The general construction begins by fixing regular cardinals $\mu<\kappa<\lambda$, where $\mu^{<\mu}=\mu, \lambda$ is supercompact, and for all $\nu<\kappa$, $\nu^{<\mu}<\kappa$. In this section let us fix such cardinals as well, but in addition we assume that $\kappa=\mu^{+}, \mu$ is supercompact, and the supercompactness of $\mu$ is indestructible under $\mu$-directed closed forcing. We also assume that for all $\alpha \geq \mu, 2^{\alpha}=\alpha^{+}$.

We define now a forcing poset $\mathbb{P}_{\lambda}$ which preserves cardinals and cofinalities less than or equal to $\mu^{+}$, collapses $\lambda$ to become $\mu^{++}$, and forces that for all regular $\theta \geq \mu^{++}$, there are stationarily many $N$ in $[H(\theta)]^{\mu^{+}}$which are internally club but not internally approachable.

We define by recursion an iterated forcing

$$
\left\langle\mathbb{P}_{i}, \dot{\mathbb{Q}}_{j}: i \leq \lambda, j<\lambda\right\rangle .
$$

For this purpose, fix a Laver function $f: \lambda \rightarrow V_{\lambda}$. So for any set $x$ and cardinal $\chi$ which is greater than or equal to $\lambda$ and the cardinality of the transitive closure of $x$, there is an elementary embedding $j: V \rightarrow M$ with critical point $\lambda$ such that $M^{\chi} \subseteq M$ and $j(f)(\lambda)=x([10])$.

Suppose $\mathbb{P}_{i}$ is defined for a fixed $i<\lambda$. If $i$ is even, let $\dot{\mathbb{Q}}_{i}$ be a $\mathbb{P}_{i}$-name for $\operatorname{ADD}(\mu)$. Suppose $i$ is odd. Let $\alpha$ be the predecessor of $i$, so $\alpha+1=i$. Let us consider two cases.

Case 1: $\alpha$ is a strongly inaccessible cardinal greater than $\mu^{+}$, for all $j<\alpha,\left|\mathbb{P}_{j}\right|<\alpha$, and $f(\alpha)$ is a regular cardinal greater than or equal to $\alpha$.

\section{Case 2: Otherwise.}

If Case 2 holds, let $\dot{\mathbb{Q}}_{i}$ be a $\mathbb{P}_{i}$-name for $\operatorname{CoLL}\left(\mu^{+}, \mu^{++}\right)$. Now suppose Case 1 holds. As an induction hypothesis, assume $\mathbb{P}_{\alpha}$ is $\alpha$-c.c.; this will follow easily from the assumptions of Case 1 and the definition of the limit stages given below. Since we use $\operatorname{CoLL}\left(\mu^{+}, \mu^{++}\right)$at unboundedly many stages below $\alpha, \mathbb{P}_{\alpha}$ forces that $\alpha$ is equal to $\mu^{++}$. Let $\theta=f(\alpha)$, which is a regular cardinal greater than or equal to $\alpha$. Since $\mathbb{P}_{\alpha}$ is $\alpha$-c.c., $\theta$ is still a regular cardinal in $V^{\mathbb{P}_{\alpha}}$ and is greater than or equal to $\alpha=\mu^{++}$.

Working in the model $V^{\mathbb{P}_{\alpha+1}}$ we define $\dot{\mathbb{Q}}_{\alpha+1}$. A condition in $\dot{\mathbb{Q}}_{\alpha+1}$ is an increasing and continuous sequence $\left\langle a_{i}: i \leq \nu\right\rangle$, where $\nu<\mu^{+}$, and for all $i \leq \nu, a_{i}$ is in $\left[H(\theta)^{V^{\mathbb{P}} \alpha}\right]^{\mu} \cap V^{\mathbb{P}_{\alpha}}$. The ordering on $\dot{\mathbb{Q}}_{\alpha+1}$ is by extension of sequences. The poset $\dot{\mathbb{Q}}_{\alpha+1}$ does not add any new $\mu$-sequences of ordinals, and adds an increasing and continuous sequence of order type $\mu^{+}$through $\left[H(\theta)^{V^{\mathbb{P} \alpha}}\right]^{\mu} \cap V^{\mathbb{P}_{\alpha}}$.

Now assume $\delta \leq \lambda$ is a limit ordinal and for all $i<\delta, \mathbb{P}_{i}$ is defined. Let $\mathbb{P}_{\delta}$ consist of all partial functions $p: \delta \rightarrow V$ such that for all $i<\delta, p \uparrow i$ is in $\mathbb{P}_{i}$, 
$|\operatorname{dom}(p) \cap \operatorname{Even}|<\mu$, and $|\operatorname{dom}(p) \cap \operatorname{Odd}|<\mu^{+}$. If $\delta$ satisfies the properties listed in Case 1, then standard arguments show that $\mathbb{P}_{\delta}$ is $\delta$-c.c.

This completes the definition. The iteration satisfies the hypotheses of Theorem 3.1 ; this is proven in more detail in Section 11 of [6]. So we can conclude that for all even $\alpha<\lambda, \mathbb{P}_{\lambda}$ factors as $\mathbb{P}_{\alpha} * \mathbb{P}_{\alpha, \lambda}$, where in $V^{\mathbb{P}_{\alpha}}$ :

(I) $\mathbb{P}_{\alpha, \lambda}$ preserves all cardinals and cofinalities less than or equal to $\mu^{+}$.

(II) $\mathbb{P}_{\alpha, \lambda}$ forces that if $h: \mu^{+} \rightarrow V^{\mathbb{P}_{\alpha}}$ is a function, all of whose initial segments are in $V^{\mathbb{P}_{\alpha}}$, then $h$ is in $V^{\mathbb{P}_{\alpha}}$.

In addition, $\mathbb{P}_{\lambda}$ is $\lambda$-c.c. and forces that $\lambda$ is equal to $\mu^{++}$. Also any bounded subset of $\lambda$ in $V^{\mathbb{P}_{\lambda}}$ is in $V^{\mathbb{P}_{i}}$ for some $i<\lambda$. Therefore $\mathbb{P}_{\lambda}$ forces that $2^{\mu}=\mu^{++}$.

Define a set $A$ by letting

$$
A=\left\{\alpha<\lambda: \mu^{+}<\alpha \wedge \alpha \text { is inaccessible } \wedge \forall i<\alpha\left|\mathbb{P}_{i}\right|<\alpha\right\} .
$$

Note that if $j: V \rightarrow M$ is an elementary embedding with critical point $\lambda$ such that $M^{\lambda} \subseteq \lambda$, then $\lambda$ is in $j(A)$.

We claim that if $\alpha$ is in $A$, then $\alpha$ has cofinality $\mu^{+}$in $V^{\mathbb{P}_{\lambda}}$. Factor $\mathbb{P}_{\lambda}$ as

$$
\mathbb{P}_{\lambda}=\mathbb{P}_{\alpha} * \operatorname{ADD}(\mu) * \dot{\mathbb{Q}} * \mathbb{P}_{\alpha+2, \lambda} .
$$

In $V^{\mathbb{P}_{\alpha}}, \alpha$ is equal to $\mu^{++}$. If $\dot{\mathbb{Q}}$ is a name for $\operatorname{CoLL}\left(\mu^{+}, \mu^{++}\right)$, then $\alpha$ has cofinality $\mu^{+}$in $V^{\mathbb{P}_{\alpha+2}}$. If $\dot{\mathbb{Q}}$ is a name for the poset for adding an increasing and continuous sequence of length $\mu^{+}$as described in Case 1 , then $\mathbb{Q}$ collapses $\mu^{++}$but does not add any $\mu$-sequences of ordinals; so $\alpha$ has cofinality $\mu^{+}$in $V^{\mathbb{P}_{\alpha+2}}$. Now $\mathbb{P}_{\alpha+2, \lambda}$ preserves the regularity of $\mu^{+}$, so $\alpha$ has cofinality $\mu^{+}$in $V^{\mathbb{P}_{\lambda}}$.

Proposition 5.1. The forcing poset $\mathbb{P}_{\lambda}$ forces that for any stationary set $B \subseteq A$, $B$ is not in $I[\lambda]$.

Proof. The proof is the same as the proof of Proposition 3.2.

Proposition 5.2. In $V^{\mathbb{P}_{\lambda}}$, for any regular cardinal $\theta \geq \mu^{++}$, there are stationarily many $N$ in $[H(\theta)]^{\mu^{+}}$such that $N \cap \lambda$ is in $A$ and $N$ is internally club but not internally approachable.

Proof. We give an overview of the proof which contains some of the essential points. For the complete details see Proposition 11.2 of [6].

Fix in $V$ an elementary embedding $j: V \rightarrow M$ with critical point $\lambda$ such that $M^{\theta} \subseteq M$ and $j(f)(\lambda)=\theta$. Consider the iteration

$$
j\left(\left\langle\mathbb{P}_{i}, \dot{\mathbb{Q}}_{i}: i<\lambda\right\rangle\right)=\left\langle\mathbb{P}_{i}^{j}, \dot{\mathbb{Q}}_{i}^{j}: i<j(\lambda)\right\rangle
$$

in $M$. Then for all $i<\lambda, \mathbb{P}_{i}^{j}=j\left(\mathbb{P}_{i}\right)=\mathbb{P}_{i}$. So in the definition of $\dot{\mathbb{Q}}_{\lambda+1}^{j}, \lambda$ satisfies Case 1 . Hence $j\left(\mathbb{P}_{\lambda}\right)$ factors as

$$
j\left(\mathbb{P}_{\lambda}\right)=\mathbb{P}_{\lambda} * \operatorname{ADD}(\mu) * \dot{\mathbb{Q}} * \mathbb{P}_{\lambda+2, j(\lambda)},
$$

where $\dot{\mathbb{Q}}$ is a name for the poset which adds an increasing and continuous sequence of order type $\mu^{+}$through $\left[H(\theta)^{M^{\mathbb{P} \lambda}}\right]^{\mu} \cap M^{\mathbb{P}_{\lambda}}$.

Let $K=G * G_{0} * G_{1} * H$ be generic for $\mathbb{P}_{\lambda} * \operatorname{ADD}(\mu) * \dot{\mathbb{Q}} * \mathbb{P}_{\lambda+2, j(\lambda)}$ over $V$. Then in the generic extension $V[K]$ we can lift $j$ to $j: V[G] \rightarrow M[K]$ such that $j(G)=K$. 
We would like to show that in $V[G]$, there are stationarily many $N$ in $[H(\theta)]^{\mu^{+}}$ such that $N \cap \lambda$ is in $A$ and $N$ is internally club but not internally approachable. So fix a function $F: H(\theta)^{<\omega} \rightarrow H(\theta)$. Since $j\left(\mu^{+}\right)=\mu^{+}$, by elementarity it suffices to show that in $M[j(G)]$, there is a set $N$ in $[j(H(\theta))]^{\mu^{+}}$such that $N \cap j(\lambda)$ is in $j(A), \mu^{+} \subseteq N, N$ is closed under $j(F)$, and $N$ is internally club but not internally approachable.

Let $N=j\left[H(\theta)^{V[G]}\right]$. Then $N$ is closed under $j(F), \mu^{+} \subseteq N$, and $N \cap j(\lambda)=$ $\lambda \in j(A)$. Let $\left\langle a_{i}: i<\mu^{+}\right\rangle$be the union of the generic $G_{1}$. Then $\left\langle j\left[a_{i}\right]: i<\mu^{+}\right\rangle$ witnesses that $N$ has size $\mu^{+}$and is internally club. Suppose for a contradiction $N$ is internally approachable. Then $H(\theta)^{V[G]}$ is internally approachable in $M[j(G)]$. Let $\left\langle M_{i}: i<\mu^{+}\right\rangle$witness that $H(\theta)^{V[G]}$ is internally approachable. Then by property (II) applied to $\mathbb{P}_{\lambda, j(\lambda)},\left\langle M_{i}: i<\mu^{+}\right\rangle$is in $M[G]$. But then $H(\theta)^{V[G]}$ has size $\mu^{+}$in $M[G]$, which is false since $\theta \geq \mu^{++}$in $M[G]$.

Lemma 5.3. The forcing poset $\mathbb{P}_{\lambda}$ is $\mu$-directed closed.

Proof. By Lemma 4.1, it suffices to show that for all $i<\lambda, \mathbb{P}_{i}$ forces that $\dot{\mathbb{Q}}_{i}$ is $\mu$-directed closed. This is clear when $\dot{\mathbb{Q}}_{i}$ is $\operatorname{ADD}(\mu)$ or $\operatorname{CoLL}\left(\mu^{+}, \mu^{++}\right)$. So assume $i=\alpha+1$, where $\alpha$ satisfies Case 1 in the definition of the iteration. Let $\theta=f(\alpha)$.

Let $G_{\alpha} * H$ be generic for $\mathbb{P}_{\alpha} * \operatorname{ADD}(\mu)$ over $V$. Working in $V\left[G_{\alpha} * H\right]$, suppose $\left\{p_{i}: i<\xi\right\}$ is a directed family of conditions in $\mathbb{Q}_{\alpha+1}$, where $\xi<\mu$. For each $i<\xi$, write $p_{i}=\left\langle a_{j}^{i}: j \leq \gamma_{i}\right\rangle$. Let $\gamma=\sup \left\{\gamma_{i}: i<\xi\right\}$, which is less than $\mu^{+}$. Given $i, k<\xi$ with $\gamma_{k} \leq \gamma_{i}$, since $p_{i}$ and $p_{k}$ are compatible and the ordering on $\mathbb{Q}_{\alpha+1}$ is by extension of sequences, it must be the case that $p_{k}$ is an initial segment of $p_{i}$. So for $j \leq \gamma_{k}, a_{j}^{k}=a_{j}^{i}$. In particular, if $\gamma=\gamma_{i}$ for some $i<\xi$, then $p_{i} \leq p_{k}$ for all $k<\xi$, and we are done. Otherwise $\bigcup\left\{p_{i}: i<\xi\right\}$ is an increasing and continuous sequence $\left\langle a_{j}: j<\gamma\right\rangle$ such that for all $i<\xi$ and $j \leq \gamma_{i}, a_{j}=a_{j}^{i}$.

Now for all $i<\gamma, a_{i}$ is in $\left[H(\theta)^{V\left[G_{\alpha}\right]}\right]^{\mu} \cap V\left[G_{\alpha}\right]$. Let $a=\bigcup\left\{a_{i}: i<\gamma\right\}$. Note that $a=\bigcup\left\{\max \left(p_{i}\right): i<\xi\right\}$. For all $i<\xi, \max \left(p_{i}\right)$ is in $V\left[G_{\alpha}\right]$. Since $\xi<\mu$ and $\operatorname{ADD}(\mu)$ is $\mu$-closed, the set $\left\{\max \left(p_{i}\right): i<\xi\right\}$ is in $V\left[G_{\alpha}\right]$. Hence $a$ is in $\left[H(\theta)^{V[G]}\right]^{\mu} \cap V\left[G_{\alpha}\right]$. Define $q: \gamma+1 \rightarrow V\left[G_{\alpha}\right]$ in $V\left[G_{\alpha+1}\right]$ by letting $q \uparrow \gamma=\left\langle a_{i}: i<\gamma\right\rangle$ and $q(\gamma)=a$. Then $q$ is a condition in $\mathbb{Q}_{\alpha+1}$ and $q \leq p_{i}$ for all $i<\xi$.

Let $G$ be a generic filter for $\mathbb{P}_{\lambda}$ over $V$, and let $W=V[G]$. Then the following facts are true in $W$. We have that $\mu$ is a supercompact cardinal, $\lambda=\mu^{++}$, and $2^{\mu}=\mu^{++}$. The set $A$ is a stationary subset of $\lambda \cap \operatorname{cof}\left(\mu^{+}\right)$, and $I\left[\mu^{++}\right]$does not contain any stationary subset of $A$. For all regular $\theta \geq \mu^{++}$, there are stationarily many $N$ in $[H(\theta)]^{\mu^{+}}$such that $N \cap \mu^{++} \in A$ and $N$ is internally club but not internally approachable.

Proposition 5.4. Let $\mathbb{P}$ be a $\mu$-centered forcing poset in $W$ which preserves $\mu$ and forces $2^{\mu}=\mu^{++}$. Then in $W^{\mathbb{P}}$, for all regular $\theta \geq \mu^{++}$, there are stationarily many $N$ in $[H(\theta)]^{\mu^{+}}$which are internally club but not internally approachable.

Proof. Since $2^{\mu}=\mu^{++}$in $W$, let $T$ be the maximal set in $I\left[\mu^{++}\right]$. Now the set $A$ has no stationary subset which is in $I\left[\mu^{++}\right]$, hence $A \cap T$ is non-stationary. Fix a club set $C \subseteq \mu^{++}$such that $A \cap T \cap C$ is empty.

By Theorem 2.1, $T$ remains the maximal set in $I\left[\mu^{++}\right]$in $W^{\mathbb{P}}$. Let $\vec{a}=\left\langle\dot{a}_{i}: i<\right.$ $\left.\mu^{++}\right\rangle$be a $\mathbb{P}$-name for an enumeration of $\left[\mu^{++}\right]^{<\mu^{+}}$. Since $T$ is the maximal set in 
the extension, there is a $\mathbb{P}$-name $\dot{D}$ for a club subset of $\mu^{++}$such that $T \cap \dot{D}=S_{\vec{a}} \cap \dot{D}$. Then

$$
\mathbb{P} \Vdash A \cap S_{\vec{a}} \cap C \cap \dot{D}=\emptyset .
$$

Let $H$ be a generic filter for $\mathbb{P}$ over $W$. In $W[H]$ let $\theta \geq \mu^{++}$be a regular cardinal. For the remainder of the proof, we write $H(\theta)$ for $H(\theta)^{W[H]}$. We will prove that there exist stationarily many $N$ in $[H(\theta)]^{\mu^{+}}$which are internally club but not internally approachable. Fix a function $F: H(\theta)^{<\omega} \rightarrow H(\theta)$. Let $\dot{F}$ be a $\mathbb{P}$-name such that $\dot{F}^{H}=F$.

Back in $W$, fix a regular cardinal $\chi$ larger than $\theta$ such that $\mathbb{P}, C, \dot{D}, \vec{a}$, and $\dot{F}$ are in $H(\chi)$. Choose a set $N$ in $[H(\chi)]^{\mu^{+}}$such that $N \cap \mu^{++}$is in $A, N$ is an elementary substructure of $H(\chi), N$ contains as elements $\mathbb{P}, C, \dot{D}, \vec{a}, \dot{F}$, and $\theta$, and $N$ is internally club but not internally approachable. Note that $\mu^{+} \subseteq N$.

Since $\mathbb{P}$ is in $H(\chi)$ and $N, H(\chi)^{W[H]}=H(\chi)^{W}[H]$ and $N[H]$ is an elementary substructure of $H(\chi)^{W[H]}$. Define

$$
N^{*}=N[H] \cap H(\theta) .
$$

We claim that $N^{*}$ is a set in $[H(\theta)]^{\mu^{+}}$such that $\mu^{+} \subseteq N^{*}, N^{*}$ is closed under $F$, and $N^{*}$ is internally club but not internally approachable.

The name $\dot{F}$ is in $N$, so $F$ is in $N[H]$, thus $N^{*}$ is closed under $F$ by elementarity. Also $\mu^{+}=N \cap \mu^{+} \subseteq N^{*}$. Since $|N[H]|=|N|,\left|N^{*}\right| \leq|N|=\mu^{+}$, and since $\mu^{+} \subseteq N^{*}, N^{*}$ has size $\mu^{+}$.

The set $N$ is internally club, so fix an increasing and continuous sequence $\left\langle N_{i}\right.$ : $\left.i<\mu^{+}\right\rangle$with union equal to $N$ such that for all $i<\mu^{+}, N_{i}$ is in $N$. We would like to show that $N^{*}$ is internally club. The sequence $\left\langle N_{i}[H] \cap H(\theta): i<\mu^{+}\right\rangle$is an increasing and continuous sequence with union $N^{*}$. Fix $i<\mu^{+}$, and we show $N_{i}[H] \cap H(\theta)$ is in $N^{*}$. Since $\theta$ is in $N[H], H(\theta)$ is in $N[H]$ by elementarity. Also $N_{i}$ and $H$ are in $N[H]$. So $N_{i}[H] \cap H(\theta)$ is in $N[H]$ by elementarity. But $N_{i}[H] \cap H(\theta)$ is a subset of $H(\theta)$ with size less than $\theta$, and hence is in $H(\theta)$. So $N_{i}[H] \cap H(\theta)$ is in $N[H] \cap H(\theta)=N^{*}$. This proves that $N^{*}$ is internally club.

Now we work towards the proof that $N^{*}$ is not internally approachable. Let $\alpha$ be equal to $N \cap \lambda$, which is in $A$. We claim that $\alpha=N^{*} \cap \lambda$ and $\alpha$ is not in $S_{\vec{a}}$. First, since $\mathbb{P}$ is $\mu^{+}$-c.c. and $\mu^{+} \subseteq N, N[H] \cap W=N$, and in particular, $\alpha=N \cap \lambda=N[H] \cap \lambda=N^{*} \cap \lambda$. Now $\dot{D}$ is in $N$, so $\dot{D}^{H}=D$ is in $N[H]$; also $C$ is in $N[H]$. So by elementarity, $\alpha$ is a limit point of $C \cap D$, and hence $\alpha$ is in $A \cap C \cap D$. Since $A \cap S_{\vec{a}} \cap C \cap D$ is empty, $\alpha$ is not in $S_{\vec{a}}$.

Let $a_{i}=\dot{a}_{i}^{H}$ for each $i<\mu^{++}$. Since $\vec{a}$ is in $N$, the sequence $\left\langle a_{i}: i<\mu^{++}\right\rangle$is in $N[H]$. In particular, $\left\{a_{i}: i<\alpha\right\}$ is a subset of $N^{*}$. Now for each $i<\alpha, a_{i}$ is a bounded subset of $\alpha$ with order type less than $\mu^{+}$. On the other hand, if $b$ is a subset of $\alpha$ in $N^{*}$ with order type less than $\mu^{+}$, then by elementarity there is $i<\alpha$ such that $b=a_{i}$. So $\left\langle a_{i}: i<\alpha\right\rangle$ is an enumeration of $N^{*} \cap[\alpha]^{<\mu^{+}}$.

Now suppose for a contradiction that $N^{*}$ is internally approachable. Let $\left\langle N_{i}^{*}\right.$ : $\left.i<\mu^{+}\right\rangle$be an increasing and continuous sequence of sets with union $N^{*}$ such that for all $\beta<\mu^{+},\left\langle N_{i}^{*}: i<\beta\right\rangle$ is in $N^{*}$. Then the set $c=\left\{\sup \left(N_{i}^{*} \cap \mu^{++}\right): i<\mu^{+}\right\}$ is a club subset of $\alpha$ with order type $\mu^{+}$. Moreover, every proper initial segment of $c$ is in $N^{*}$ since it is definable in $N^{*}$ from $\mu^{++}$and a proper initial segment of $\left\langle N_{i}^{*}: i<\mu^{+}\right\rangle$. But $\left\{a_{i}: i<\alpha\right\}=N^{*} \cap[\alpha]^{<\mu^{+}}$, so every proper initial segment of $c$ is in $\left\{a_{i}: i<\alpha\right\}$. This implies $\alpha$ is in $S_{\vec{a}}$, which is a contradiction. 
All of the posets we considered in Section 4 for singularizing $\mu$ preserve $\mu$, are $\mu$-centered, and have size $2^{\mu}=\mu^{++}$in $W$. This implies that such posets force $2^{\mu}=\mu^{++}$over $W$. Theorem 3 now follows from our construction of $W=V[G]$ in this section, together with Proposition 5.4, by the same arguments as given in Section 4 .

\section{REFERENCES}

[1] J. Baumgartner. Iterated forcing. In Surveys in Set Theory, pages 1-59. Cambridge Univ. Press, 1983.

[2] J. Cummings. Notes on singular cardinal combinatorics. Notre Dame J. Formal Logic, 46(3):251-282, 2005.

[3] M. Foreman and S. Todorčević. A new Löwenheim-Skolem theorem. Trans. Amer. Math. Soc., 357:1693-1715, 2005.

[4] M. Foreman and H. Woodin. The G.C.H can fail everywhere. Ann. of Math., 133:1-35, 1991.

[5] T. Jech. Set Theory. Springer, 1997. Second edition.

[6] J. Krueger. Some applications of mixed support iterations. To appear in the Annals of Pure and Applied Logic.

[7] J. Krueger. Internally club and approachable. Adv. Math., 213(2):734-740, 2007.

[8] J. Krueger. A general Mitchell style iteration. Math. Log. Q., 54(6):641-651, 2008.

[9] J. Krueger. Internally club and approachable for larger structures. Fund. Math., 201:115-129, 2008.

[10] R. Laver. Making the supercompactness of $\kappa$ indestructible under $\kappa$-directed closed forcing. Israel J. Math., 29(4):385-388, 1978.

[11] M. Magidor. On the singular cardinals problem I. Israel J. Math., 28:1-31, 1977.

[12] M. Magidor. Changing cofinality of cardinals. Fund. Math., 99:61-71, 1978.

[13] W. Mitchell. Aronszajn trees and the independence of the transfer property. Ann. Math. Logic, 5:21-46, 1972/73.

[14] L.B. Radin. Adding closed cofinal sequences to large cardinals. Ann. Math. Logic, 22:243-261, 1982.

[15] S. Shelah. On successors of singular cardinals. In M. Boffa, D. van Dalen, and K. McAloon, editors, Logic Colloquium 78, pages 357-380. North Holland Publishing Company, 1979.

[16] S. Shelah. Reflecting stationary sets and successors of singular cardinals. Arch. Math. Logic, 31(1):25-53, 1991.

School of Mathematical Sciences, Tel Aviv University

E-mail address: gitik@post.tau.ac.il

URL: http://www.math.tau.ac.il/ gitik

Department of Mathematics, University of California, Berkeley

E-mail address: jkrueger@math.berkeley.edu

URL: http://www.math. berkeley.edu/ jkrueger 